\title{
Observing the WHIM with Athena
}

\author{
T. Brand ${ }^{\mathrm{a}}$, J. Wilms ${ }^{\mathrm{a}}$, T. Dauser ${ }^{\mathrm{a}}$, P. Peille ${ }^{\mathrm{b}}$, B. Cobo ${ }^{\mathrm{c}}$, M. Ceballos ${ }^{\mathrm{c}}$, R.A. den Hartog ${ }^{\mathrm{d}}$, \\ J.S. Kaastra ${ }^{\mathrm{d}}$, P. O'Brien ${ }^{\mathrm{e}}$, X. Barcons ${ }^{\mathrm{c}}$, L. Piro ${ }^{\mathrm{f}}$, J.-W.A. den Herder $^{\mathrm{d}}$, and D. Barret ${ }^{\mathrm{b}}$ \\ ${ }^{a}$ Dr. Remeis-Sternwarte and Erlangen Centre for Astroparticle Physics, Universität \\ Erlangen-Nürnberg, Sternwartstr. 7, 96049 Bamberg, Germany \\ ${ }^{\mathrm{b}}$ Institut de Recherche en Astrophysique et Planétologie, 9 avenue du Colonel Roche, \\ BP 44346, 31028 Toulouse Cedex 4, France \\ 'Instituto de Física de Cantabria, Edificio Juan Jorda, Campus Universidad de Cantabria, \\ Avda. de los Castros s/n, 39005 Santander, Spain \\ ${ }^{\mathrm{d} S R O N}$ Netherlands Institute for Space Research, Sorbonnelaan 2, 3584 CA Utrecht, \\ Netherlands \\ eUniversity of Leicester, University Road, Leicester, LE1 7RH, United Kingdom \\ ${ }^{\mathrm{f}}$ Istituto Nazionale di Astrofisica - Istituto di Astrofisica e Planetologia Spaziali, Via Fosso del \\ Cavaliere 100, Tor Vergata, 00133 Roma, Italy
}

\begin{abstract}
We present simulations of the detection probability for absorption lines from ions in the warm and hot ionized medium (WHIM) with Athena in the spectra of Gamma-ray burst afterglows. The simulations are based on Swift XRT lightcurves of these afterglows and are performed using the end-to-end simulation framework SIXTE. We simulate both the case of single and multiple absorption lines, as well as results for line searches in absorption structures from a more complex medium. We show that the Athena X-IFU can detect WHIM lines with strong OvII lines (equivalent widths larger than $0.14 \mathrm{eV}$ ) in spectra containing $3 \times 10^{6}$ counts.
\end{abstract}

Keywords: Athena, X-IFU, GRB, WHIM, Simulation, SIXTE

\section{INTRODUCTION}

One of the science goals of the Athena observatory is to find missing baryons in the intergalactic medium up to a redshift of $z=2$ [1]. A part of these baryons is expected to be present in the warm hot intergalactic medium (WHIM). These filaments do not radiate enough to be observable by their own light, but need to be measured by the absorption of background radiation [2]. This requires a bright source at a large distance. The Athena Mission Proposal sets the goal to detect filaments against 25 AGN and 40 Gamma-ray bursts (GRBs, [3]). In this paper we examine the detection efficiency of the WHIM with the X-IFU using GRB afterglows.

In order to investigate the observability of the WHIM and to give predictions on the quality of these observations, we undertake simulations with the SIXTE framework, the official simulation framework for the Athena WFI and X-IFU detectors* [4]. The end product of these simulations are event files which can be analysed using common X-ray data analysis tools (e.g., [5]).

The remainder of this contribution is structured as follows. In Section 2 we give a description of the simulations. We discuss the properties of the simulated spectra and our results in Section 3. Finally, in Section 4 we discuss the consequences of the simulations for Athena.

Further author information: (Send correspondence to T. Brand)

T. Brand: E-mail: thorsten.brand@fau.de

J. Wilms E-mail: joern.wilms@sternwarte.uni-erlangen.de

*http://www.sternwarte.uni-erlangen.de/research/sixte/

Space Telescopes and Instrumentation 2016: Ultraviolet to Gamma Ray, edited by Jan-Willem A. den Herder, Tadayuki Takahashi, Marshall Bautz, Proc. of SPIE Vol. 9905, 99055F - (c) 2016 SPIE · CCC code: 0277-786X/16/\$18 - doi: 10.1117/12.2234442 

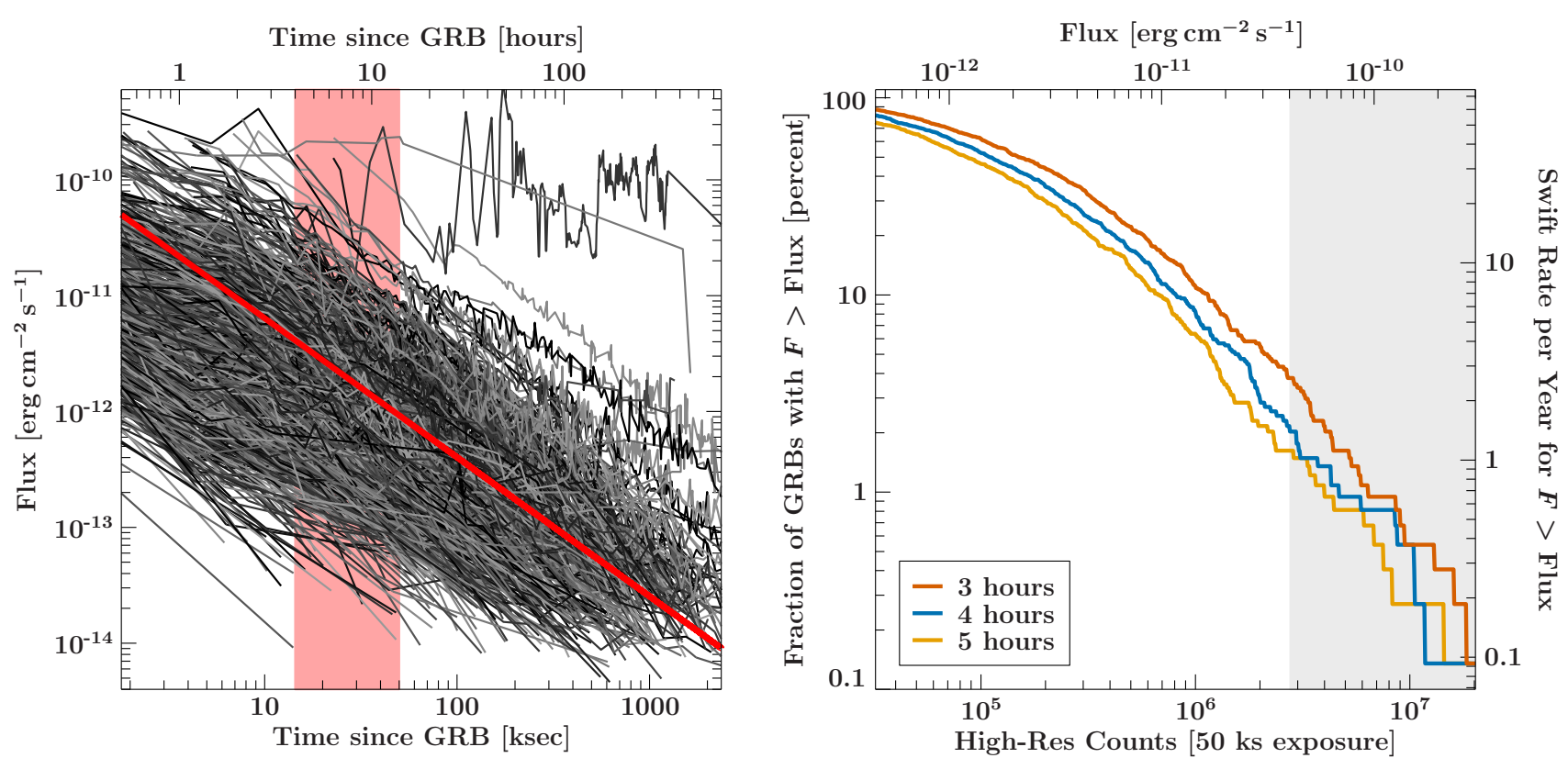

Figure 1. Left: All Swift GRB lightcurves measured between 2004 December 18 until 2015 September 31 (948 in total). We find an approximation for the light curve of $F \propto t^{-1.2}$ (red line). The red shaded region depicts the time regime which is of interest to our simulations. Right: Cumulative number of GRB afterglows as found by Swift for three reference times after the initial outburst. The lower $x$-axis gives the equivalent number of high-resolution counts for a $50 \mathrm{ks}$ observation, the top $x$-axis gives the $0.3-10 \mathrm{keV}$ flux at the start of the observation. Shaded in grey is the flux region in which single lines can be reconstructed with a false alarm probability less than $20 \%$ for EWs larger than 0.14 eV. (Sect. 2.4).

\section{DESCRIPTION OF THE SIMULATIONS}

WHIM filaments imprint faint absorption lines in the X-ray spectra of background sources. The Athena X-IFU is designed to resolve spectral features with a full width at half maximum of $2.5 \mathrm{eV}$ to find a larger number of these filaments. We use SIXTE with its Athena X-IFU setup to investigate the effective capability of the current instrument baseline to detect the WHIM absorption lines. In the following subsections we present the assumptions made in our simulations.

\subsection{SWIFT LIGHTCURVES AND FLUX RANGE}

The detection of faint absorption lines is obviously dependent on the signal to noise ratio of the underlying X-ray spectrum. For GRB afterglows, only a limited amount of time is available before the afterglow becomes too faint to be suitable for WHIM detection. To obtain a realistic model for the temporal behavior of the source flux we analyse all available Swift GRB afterglow observations from http://www.swift.ac.uk/xrt_curves/ [6, 7] until 31 September 2015 in the $0.3-10 \mathrm{keV}$ energy band (Fig. 1). As also found by Margutti et al. [8] we find a powerlaw with an index of -1.2 to represent most light curves well (red line in Fig. 1). Such a power law model, together with a model spectrum, can be used to find the connection between the flux of the afterglow at the start of the observation and the number of counts collected by X-IFU for a given exposure time. Note that due to the strong decrease in flux, half of the counts of a $50 \mathrm{ks}$ exposure are observed in the first $14 \mathrm{ks}$ of the observation. It is therefore very important to trigger observations as early as possible after an outburst was detected. In the following we assume the start of an X-IFU observation at 4 hours after the GRB outburst. This time interval is the baseline TOO reaction time of Athena [1].

In transition edge sensor detectors such as the X-IFU the temperature change in the device caused by the absorption of a photon is used to determine the photon's energy. The precision with which this energy can be reconstructed depends on the time difference between the previous, the current, and the following photon, and is thus flux dependent (see Peille et al., these proceedings [9], for a description of possible event reconstruction 
Table 1. Spectral parameters for the GRB afterglow and WHIM model.

\begin{tabular}{lrr}
\hline Parameter & Value & Reference \\
\hline \hline GRB Afterglow & & \\
Photon Index & 2.0 & {$[10]$} \\
Redshift & 2.0 & {$[8]$} \\
Intrinsic Absorption & & \\
\hline WHIM O VII Line at $\mathbf{5 7 4} \mathbf{~ e V}$ & $0.0-0.1$ & \\
Redshift & $0.07-0.28 \mathrm{eV}$ & {$[10] ;$ E. Ursino, priv. comm. } \\
Equivalent Width & $0.1 \mathrm{eV}$ & \\
$\sigma$ & optional & \\
\hline WHIM O viII Line at $\mathbf{6 5 4} \mathbf{~ e V}$ & $2 / 3 \mathrm{of} \mathrm{O} \mathrm{vII}$ & \\
Equivalent width & $0.1 \mathrm{eV}$ & \\
$\sigma$ & $2 \times 10^{20} \mathrm{~cm}^{-2}$ & \\
\hline Galactic Absorption &
\end{tabular}

methods). In our simulations, we classify events into high-, medium-, and low-resolution events. Only highresolution events, for which the energy is reconstructed to the nominal resolution of the device (2.5 eV), are the used for the following analysis. The percentage of medium- and low-resolution events is, in the critical flux regime, smaller than $1 \%$.

The Swift data are also used to determine the relevant range for the starting flux of the GRB afterglows. Figure 1 shows the cumulative number of GRB afterglows found by Swift and motivates the relevant flux regime. At this time, the $0.3-10 \mathrm{keV}$ XRT band flux lies between $10^{-12}$ and $10^{-10} \mathrm{erg} \mathrm{cm}^{-2} \mathrm{~s}^{-1}$. In total numbers, Swift finds about two GRB-afterglows per year in its field of view which are brighter than 1 mCrab at four hours after the initial outburst.

\subsection{SOURCE MODEL}

The spectral shape of all simulated GRBs consists of a powerlaw with photon index $\Gamma=2$, which is absorbed by the host galaxy and our own Galaxy $[7,8,10]$. As discussed above, the flux of the source varies as $F \propto t^{-1.2}$. The model parameters are given in detail in Table 1. For the absorption models we use vern cross sections [11] and wilm abundances [12].

In our simulations we describe the WHIM filaments through their strongest lines the the $\mathrm{O}$ VII line at $574 \mathrm{eV}$ and the $\mathrm{O}$ VIII line at $654 \mathrm{eV}$. Both have equivalent widths smaller than $0.28 \mathrm{eV}$ (for expected ranges of the equivalent width see, e.g., Branchini et al. [10]). In the case of two lines, the equivalent width of the O VIII line is fixed to $2 / 3$ of the $\mathrm{O}$ VII line in the input source model [10].

\subsection{INSTRUMENT SETUP}

For all simulations presented in the following, we use the current baseline model of the X-IFU Small Pixel Array (SPA) together with the baseline mirror, which has an outer radius of $1469 \mathrm{~mm}$. The filter setup is assumed to consist of 5 individual filters with a total of $280 \mathrm{~nm}$ Polyimide and $210 \mathrm{~nm}$ Al. Additionally, the two outermost filters have a support grid of Polymide with a thickness of $10 \mu \mathrm{m}$ and an open area fraction of 0.93 . The result is an effective area of about $1.5 \mathrm{~m}^{2}$ at $1 \mathrm{keV}$. The source and detector models as described above are used with SIXTE to create an event file for each virtual observation. The start of each observation is at 4 hours after the initial outburst. We examine observations with an exposure of $50 \mathrm{ks}$. This is the baseline exposure time of the X-IFU for TOOs [13].

\subsection{DESCRIPTION OF THE ANALYSIS}

For the analysis of the simulated event files the spectrum is rebinned to a bin width of $0.8 \mathrm{eV}$, oversampling the spectral resolution of the X-IFU by a factor of three. In the critical flux range we find several hundreds of counts in each bin and therefore use $\chi^{2}$-statistics for spectral fitting. The X-ray spectrum is modeled by a power law continuum which is modified by Galactic absorption and absorption within the GRB's host galaxy. We assume that the Galactic absorption as well as the redshift of the GRB's host galaxy is known from other observations. 
In the case of simulations of two WHIM absorption lines we fix the energy distance between these two lines in our fit model to the literature value (see above). The equivalent widths of the two lines are not linked to each other, as they depend on the unknown density and ionization structure of the absorber. The photon index, the flux normalization constant, the intrinsic absorption, and the line widths are the remaining free parameters.

A blind line search with $0.0 \leq z \leq 0.1$ is used to determine the most significant line position in the spectrum. This limited redshift range is later extended to $z \leq 0.5$. Details of our search strategy are described by Protassov et al. [14]: We discretize the search energy range in steps smaller than the energy resolution of the instrument (we choose $\Delta E=0.5 \mathrm{eV}$ for the single line model and $\Delta z=1.4 \times 10^{-3}$ for the two line model). At each of these energies, we fit a narrow line to the data. We then posit the presence of a line at the minimum of the energy dependent fit statistics. The significance of this feature is then determined by a Monte Carlo simulation for which 100 realizations of the best-fit continuum model without a line are generated and subjected to the same blind line search as before. The line found in the original data set is accepted to be correct if the $\chi^{2}$-value of the original fit is smaller than $90 \%$ of the $\chi^{2}$-values of the trial fits to spectra that do not contain a line.

To measure detection probabilities, we repeat the simulation and analysis for each combination of the starting flux and the equivalent width 51 times. In each of these simulations, the redshift of the WHIM filament was varied randomly between $z=0.0$ and 0.1 . We consider a line to be correctly identified if the $90 \%$ confidence interval of the line energy contains the original line enery. Additionally we accept cases in which the difference in line energy is smaller than $1.25 \mathrm{eV}$ in the case of the single line input spectrum or $\Delta z \leq 1 \times 10^{-3}$ in the case of two lines.

\section{RESULTS}

In order to study the line detection capability of the X-IFU we define two measures for the detection accuracy: The detection probability and the false alarm probability:

- The detection probability is defined as the empirical probability that a line or line pair is found with a significance of more than $90 \%$ and that its redshift is accepted to be correct within the margins explained in Sect. 2.4. This number indicates the minimal number of counts necessary to detect absorption lines with a given strength.

- The false alarm probability gives the probability that the line position is reconstructed incorrectly in terms of the definition but is flagged as significant by the analysis algorithm. This number is important because it predicts the probability with which spurious noise pattern are found to be more significant than the input line. These false detections need to be carefully treated, as they might wrongly influence the results obtained from the spectral analysis of this WHIM filament.

In order to highlight the strengths and limitations of the WHIM measurments, we undertake two different tests: First we compare simulations with only one input line to simulations with two input lines to quantify the benefits of searching for a line pattern instear of a single absorption line. This is thought to improve the detection efficiency as we expect to find the signature of the filament in a larger number of energy bins.

We examine the simulations for four different line strengths on the relevant flux range. The flux dependent detection probability (Fig. 2) shows that the detection efficiency increases as we measure more counts from the GRB afterglow. Furthermore, a factor of four in the equivalent width between the faintest and weakest of the lines simulated has the same effect on the detection probability as a change of one order in magnitude of the starting flux. The faintest line with $0.07 \mathrm{eV}$ is at the detection limit of the X-IFU and can hardly be detected efficiently in the brightest afterglows. The stronger lines are found efficiently to $60-100 \%$ for starting fluxes higher than $4 \times 10^{-11} \mathrm{erg} \mathrm{cm}^{-2} \mathrm{~s}^{-1}$. Adding a second line to the input spectrum and the analysis increases the effective number of bins in which we can find the feature, and clearly improves the detectability of the WHIM filament systematically, if only a smaller amount. It is roughly equivalent to a raise of $15 \%$ in the detection probability for most of the probed parameter combinations.

In Fig. 3 the false alarm probability is shown for the same parameter combinations. The lower the number of counts we get from the afterglow, the more false detections are found. For a starting flux smaller than $2 \times 10^{-12} \mathrm{erg} \mathrm{cm}^{-2} \mathrm{~s}^{-1}$ we find that one half of all detections are confusions with noise even for the most prominent lines with an equivalent width of $0.28 \mathrm{eV}$. For the weakest lines this fraction is reached already at a flux of 
$\mathrm{X}$-IFU high res counts for $50 \mathrm{ks}$ exposure $[0.3-10] \mathrm{keV}$

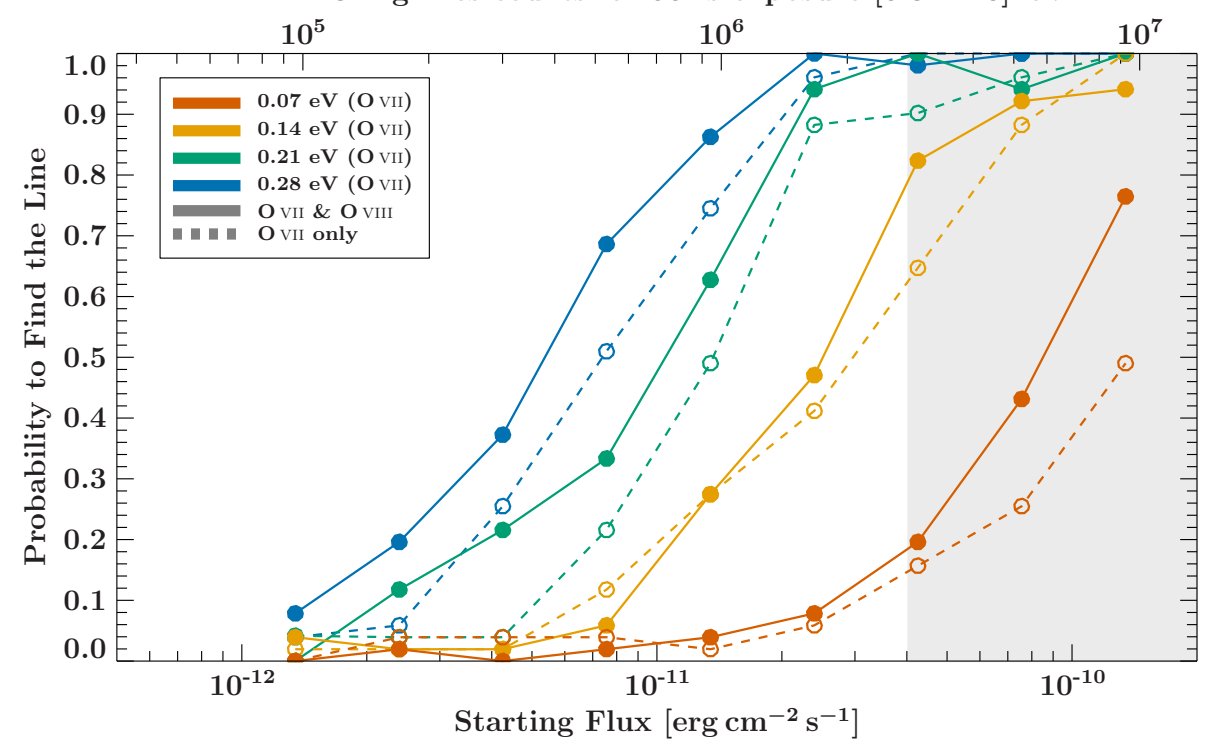

Figure 2. Detection probability for one and two input lines as function of the starting flux for different line strengths.

X-IFU high res counts for $50 \mathrm{ks}$ exposure $[0.3-10] \mathrm{keV}$

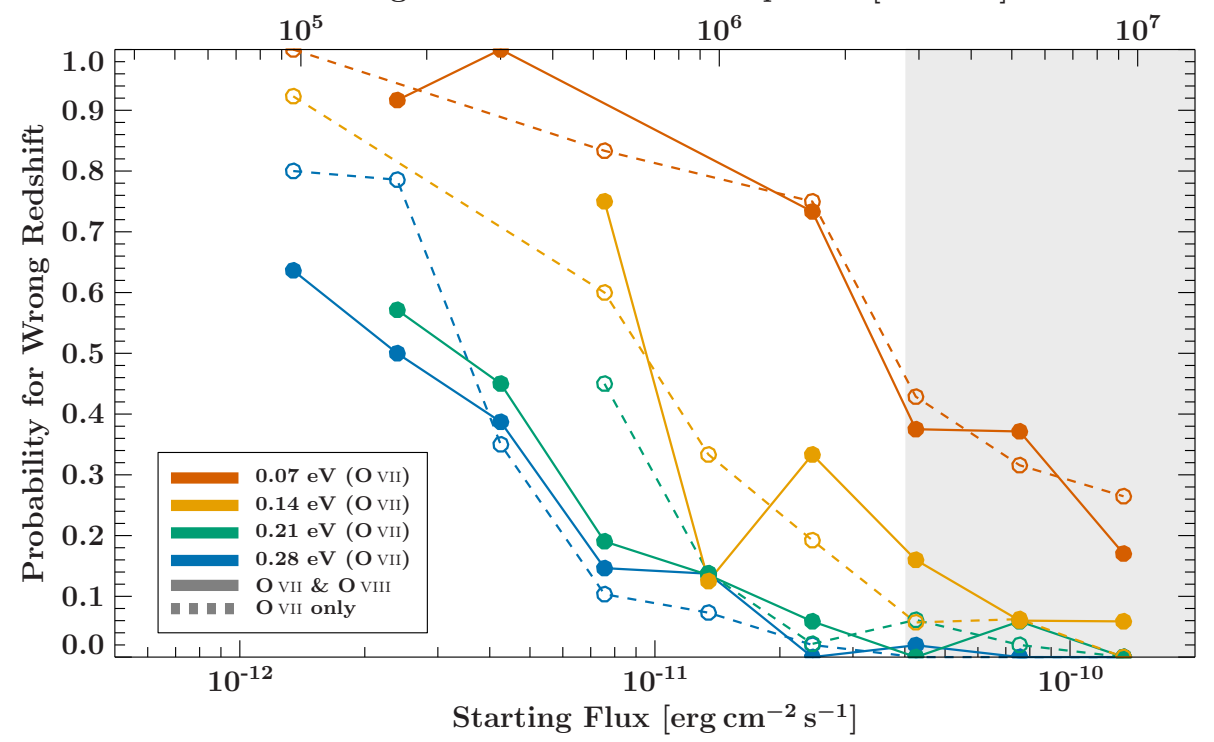

Figure 3. False alarm probability for one and two input lines as function of the starting flux for different line strengths. Note that the sample size for each data point is smaller than the total number of simulations, as we only take the runs into account for which a significant line was found. Therefore only data points with more than 10 samples are shown. 
$\mathrm{X}$-IFU high res counts for $50 \mathrm{ks}$ exposure $[0.3-10] \mathrm{keV}$

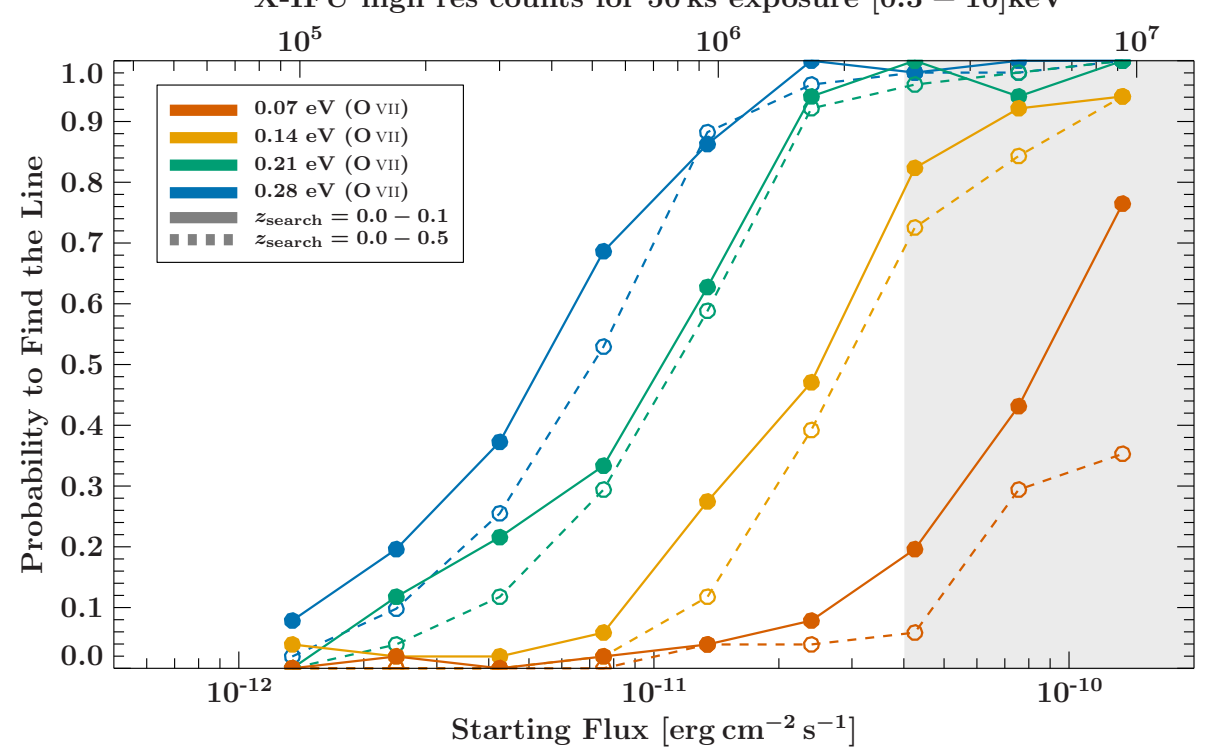

Figure 4. Detection probability for two input lines as function of the starting flux for different line strengths as before. Here an extended search region of $z \leq 0.5$ is compared to the results for a limit of $z=0.1$.

$\mathrm{X}$-IFU high res counts for $50 \mathrm{ks}$ exposure $[0.3-10] \mathrm{keV}$

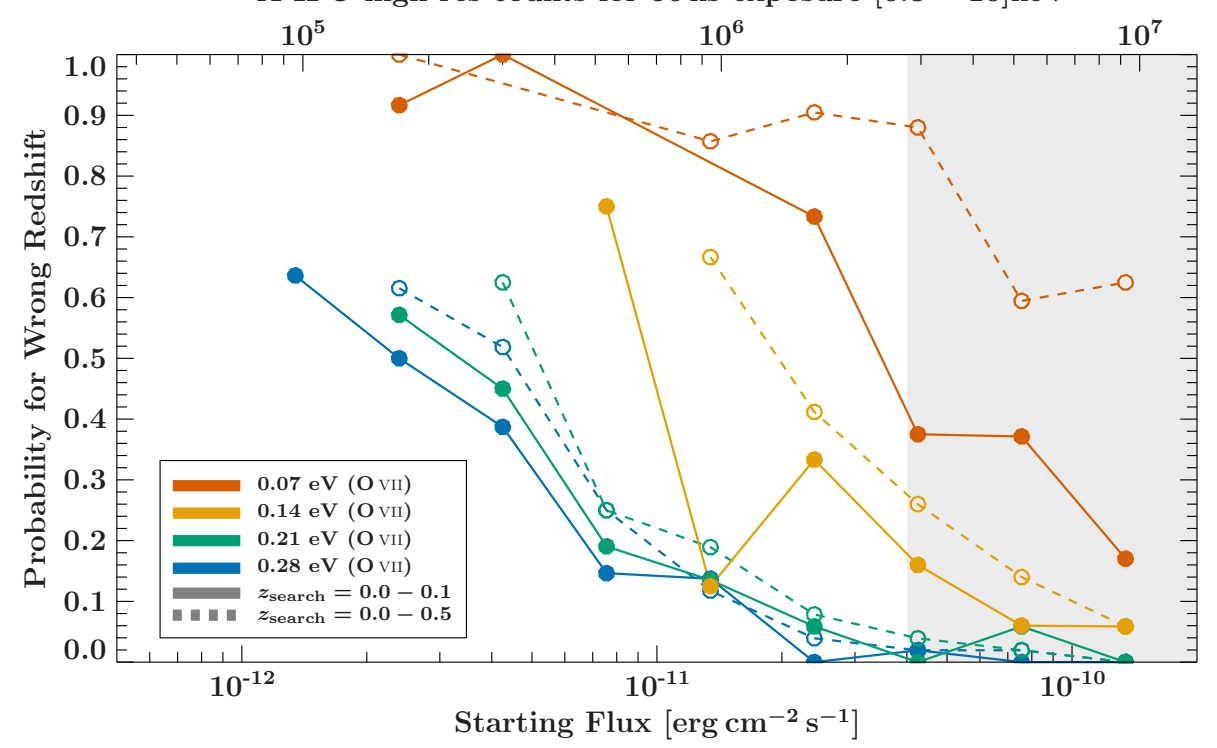

Figure 5. False alarm probability for two input lines as function of the starting flux for different line strengths. The extended search region up to $z=0.5$ is compared to the previous results for a limit of $z=0.1$.

$4 \times 10^{-11} \mathrm{erg} \mathrm{cm}^{-2} \mathrm{~s}^{-1}$. For the lines stronger than $0.14 \mathrm{eV}$ the false alarm probability at this brightness is in the order of $10 \%$ and below.

As a second test we perform the same simulations as we did for the two line case, but now extend the search region to $z \leq 0.5$ (Figs. 4 and 5). The benefit of using two absorption features instead of a single line almost completely vanishes for the extended search region - we measure a degradation in the order of $15 \%$ in the detection probability. The reason for this behavior is that a larger energy range increases the likelihood that random noise is confused with real absorption features. This behavior is also seen when considering the false alarm probability, which is significantly higher for lines weaker than $0.21 \mathrm{eV}$ when extending the search range. Effectively this means that for the $0.14 \mathrm{eV}$ line the false alarm probability almost doubles for starting fluxes 
higher than $4 \times 10^{-11} \mathrm{erg} \mathrm{cm}^{-2} \mathrm{~s}^{-1}$. For the remaining lines, the false alarm probability at this brightness still seems to be acceptable $(<10 \%)$, but with a quick degradation towards lower fluxes. This effect complicates the search for the WHIM filaments which shall be found up to $z=2$ with the X-IFU [1].

\section{CONCLUSIONS}

In the previous sections we presented the Swift GRB afterglow sample and simulations of WHIM absorption line observations with the X-IFU and demonstrate the results of a blind line search algorithm. We find that lines with equivalent widths of $0.14 \mathrm{eV}$ and brighter can be found with high probability in GRB afterglows with a starting flux of $4 \times 10^{-11} \mathrm{erg} \mathrm{cm}^{-2} \mathrm{~s}^{-1}$.

We show the impact of using two absorption lines versus a single line. The benefit from this extension is an increase of roughly $15 \%$ in terms of detection probability in the critical regime. The false alarm probability does not change significantly with respect to the small statistical sample. However, these data are produced with a blind line search interval of only $\Delta z=0.1$. This is only a small part of the redshift range in which WHIM filaments are to be found. We compare these simulations to an extended analysis up to $z=0.5$ and find a degradation of again about $15 \%$ in the critical regime in the detection probability while using two absorption lines. We also find a comparable degradation of the false alarm probability for an equivalent width of the O vII line of $0.14 \mathrm{eV}$ and an even higher degradation of approximately $50 \%$ for the weakest line simulated with a strength of $0.07 \mathrm{eV}$.

The combined picture leads us to the conclusion that we expect a limit of the starting flux of the GRB afterglow at the time of the observation of around $4 \times 10^{-11} \mathrm{erg} \mathrm{cm}^{-2} \mathrm{~s}^{-1}$ down to which we can effectively detect WHIM filaments with the strong O VII line more than $0.14 \mathrm{eV}$. This limit holds for an exposure time of the X-IFU limit of $50 \mathrm{ks}$ and is equivalent to approximately $3 \times 10^{6} \mathrm{X}$-IFU high resolution counts.

The distribution of the Swift BAT afterglow sample gives us a rate of approximately two GRBs with a starting flux above the said threshold per year. As the field of view of the BAT instrument is $1.4 \mathrm{sr}$ [15], on average we expect 9-18 GRBs per year on the full sky which can be used to detect WHIM filaments reliably with Athena.

\section{ACKNOWLEDGMENTS}

This work made use of data supplied by the UK Swift Science Data Centre at the University of Leicester and of ISIS functions (ISISscripts) provided by ECAP/Remeis observatory and MIT (http://www. sternwarte. uni-erlangen.de/isis/). We thank J.E. Davis for the development of the SLXfig module, which was used to create all figures in this paper. We acknowledge funding by the Deutsches Zentrum für Luft- und Raumfahrt contract 50 QR 1402.

\section{References}

[1] Nandra, K., Barret, D., Barcons, X., Fabian, A., den Herder, J.-W., Piro, L., Watson, M., Adami, C., Aird, J., Afonso, J. M., and et al., "The Hot and Energetic Universe: A White Paper presenting the science theme motivating the Athena+ mission," arXiv:1306.2307 (June 2013).

[2] Nicastro, F., Elvis, M., Krongold, Y., Mathur, S., Gupta, A., Danforth, C., Barcons, X., Borgani, S., Branchini, E., Cen, R., Davé, R., Kaastra, J., Paerels, F., Piro, L., Shull, J. M., Takei, Y., and Zappacosta, L., "Chandra View of the Warm-hot Intergalactic Medium toward 1ES 1553+113: Absorption-line Detections and Identifications. I.," Astrophys. J. 769, 90 (June 2013).

[3] Nandra, K., Barcons, X., den Herder, J.-W., Watson, M., Barret, D., Fabian, A., Piro, L., et al., "Athena: The Advanced Telescope for High Energy Astrophysics: A mission addressing The Hot and Energetic Universe science theme," http://www.the-athena-x-ray-observatory.eu/images/AthenaPapers/The Athena_Mission_Proposal.pdf (2014).

[4] Wilms, J., Brand, T., Barret, D., Beuchert, T., den Herder, J.-W., Kreykenbohm, I., Lotti, S., Meidinger, N., Nandra, K., Peille, P., Piro, L., Rau, A., Schmid, C., Smith, R. K., Tenzer, C., Wille, M., and Willingale, R., "ATHENA end-to-end simulations," in [Space Telescopes and Instrumentation 2014: Ultraviolet to Gamma Ray], Takahashi, T., den Herder, J.-W. A., and Bautz, M., eds., Proc. SPIE 9144, 91445 (July 2014). 
[5] Houck, J. C. and Denicola, L. A., "ISIS: An Interactive Spectral Interpretation System for High Resolution X-Ray Spectroscopy," in [Astronomical Data Analysis Software and Systems IX], N. Manset, C. Veillet, \& D. Crabtree, ed., Astronomical Society of the Pacific Conference Series 216, 591 (2000).

[6] Evans, P. A., Beardmore, A. P., Page, K. L., Tyler, L. G., Osborne, J. P., Goad, M. R., O’Brien, P. T., Vetere, L., Racusin, J., Morris, D., Burrows, D. N., Capalbi, M., Perri, M., Gehrels, N., and Romano, P., "An online repository of Swift/XRT light curves of $\gamma$-ray bursts," Astron. Astrophys. 469, 379-385 (July 2007).

[7] Evans, P. A., Beardmore, A. P., Page, K. L., Osborne, J. P., O’Brien, P. T., Willingale, R., Starling, R. L. C., Burrows, D. N., Godet, O., Vetere, L., Racusin, J., Goad, M. R., Wiersema, K., Angelini, L., Capalbi, M., Chincarini, G., Gehrels, N., Kennea, J. A., Margutti, R., Morris, D. C., Mountford, C. J., Pagani, C., Perri, M., Romano, P., and Tanvir, N., "Methods and results of an automatic analysis of a complete sample of Swift-XRT observations of GRBs," Monthly Not. Royal Astron. Soc. 397, 1177-1201 (Aug. 2009).

[8] Margutti, R., Zaninoni, E., Bernardini, M. G., Chincarini, G., Pasotti, F., Guidorzi, C., Angelini, L., Burrows, D. N., Capalbi, M., Evans, P. A., Gehrels, N., Kennea, J., Mangano, V., Moretti, A., Nousek, J., Osborne, J. P., Page, K. L., Perri, M., Racusin, J., Romano, P., Sbarufatti, B., Stafford, S., and Stamatikos, M., "The prompt-afterglow connection in gamma-ray bursts: a comprehensive statistical analysis of Swift X-ray light curves," Monthly Not. Royal Astron. Soc. 428, 729-742 (Jan. 2013).

[9] Peille, P., Ceballos, M. T., Cobo, B., Wilms, J., Bandler, S., Smith, S. J., Dauser, T., Brand, T., de Plaa, J., Barret, D., den Herder, J.-W., Piro, L., Barcons, X., and Pointecouteau, E., "Performance assessment of different pulse reconstruction algorithms for the Athena X-ray Integral Field Unit," in [Space Telescopes and Instrumentation 2016: Ultraviolet to Gamma Ray], Proc. SPIE 9905 (2016). these proceedings.

[10] Branchini, E., Ursino, E., Corsi, A., Martizzi, D., Amati, L., den Herder, J. W., Galeazzi, M., Gendre, B., Kaastra, J., Moscardini, L., Nicastro, F., Ohashi, T., Paerels, F., Piro, L., Roncarelli, M., Takei, Y., and Viel, M., "Studying the Warm Hot Intergalactic Medium with Gamma-Ray Bursts," Astrophys. J. 697, 328-344 (May 2009).

[11] Verner, D. A., Ferland, G. J., Korista, K. T., and Yakovlev, D. G., "Atomic Data for Astrophysics. II. New Analytic FITS for Photoionization Cross Sections of Atoms and Ions," Astrophys. J. 465, 487 (July 1996).

[12] Wilms, J., Allen, A., and McCray, R., "On the Absorption of X-Rays in the Interstellar Medium," Astrophys. J. 542, 914-924 (Oct. 2000).

[13] Barret, D., den Herder, J.-W. A., Piro, L., Trong, T. L., Barcons, X., et al., "The Athena x-ray integral field unit," in [Space Telescopes and Instrumentation 2016: Ultraviolet to Gamma Ray], Proc. SPIE 9905 (2016). these proceedings.

[14] Protassov, R., van Dyk, D. A., Connors, A., Kashyap, V. L., and Siemiginowska, A., "Statistics, Handle with Care: Detecting Multiple Model Components with the Likelihood Ratio Test," Astrophys. J. 571, 545-559 (May 2002).

[15] Barthelmy, S. D., Barbier, L. M., Cummings, J. R., Fenimore, E. E., Gehrels, N., Hullinger, D., Krimm, H. A., Markwardt, C. B., Palmer, D. M., Parsons, A., Sato, G., Suzuki, M., Takahashi, T., Tashiro, M., and Tueller, J., "The Burst Alert Telescope (BAT) on the SWIFT Midex Mission," Space Sci. Rev. 120, 143-164 (Oct. 2005). 